\title{
Probing the interior of asteroid Apophis: a unique opportunity in 2029
}

\author{
Patrick Michel $^{1}$, J. Y. Prado ${ }^{2}$, M. A. Barucci ${ }^{3}$, O. Groussin ${ }^{4}$,

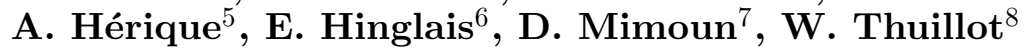 \\ and D. Hestroffer ${ }^{9}$ \\ ${ }^{1}$ Laboratoire Lagrange, University of Nice Sophia-Antipolis, Côte d'Azur Observatrory \\ BP 4229, 06304 Nice Cedex 4, France \\ email: michelp@oca.eu \\ ${ }^{2} \mathrm{CNES}$ \\ 18 Avenue Edouard Belin, 31401 Toulouse Cedex 9, France \\ email: jean-yves.prado@cnes.fr \\ ${ }^{3}$ LESIA, Paris Observatory \\ 5 Place Jules Janssen, 92195 MEUDON Cedex, France \\ email: antonella.barucci@obspm.fr \\ ${ }^{4}$ Laboratoire d'Astrophysique de Marseille \\ Technopole de Marseille-Etoile, 38 rue Frdric Joliot-Curie, 13388 Marseille Cedex 13, France \\ email: olivier.groussin@oamp.fr \\ ${ }^{5}$ Institut de Plantologie et d'Astrophysique de Grenoble - IPAG \\ Bat D de Physique, BP. 53, 38041 Grenoble, France \\ email: alain.herique@obs.ujf-grenoble.fr \\ ${ }^{6} \mathrm{CNES}$ \\ 18 Avenue Edouard Belin, 31401 Toulouse Cedex 9, France \\ email: emmanuel.hinglais@cnes.fr \\ ${ }^{7}$ Institut Suprieur de l'Aronautique et de l'Espace (ISAE) \\ 10 avenue Edouard Belin, BP 54032, 31055 Toulouse cedex 4, France \\ email: david.mimoun at isae.fr \\ ${ }^{8} \mathrm{IMCCE}$ \\ UMR 8028 CNRS, Observatoire de Paris, 77 Av. Denfert Rochereau, 75014 Paris, France \\ email: william.thuillot@imcce.fr \\ ${ }^{9} \mathrm{IMCCE}$ \\ UMR 8028 CNRS, Observatoire de Paris, 77 Av. Denfert Rochereau, 75014 Paris, France \\ email: Daniel.Hestroffer@imcce.fr
}

\begin{abstract}
The near Earth asteroid (99942) Apophis, discovered in 2004, (with a diameter of about 270 meters) will come back very close to the Earth on April 13, 2029.

The close approach of Apophis to the Earth in 2029 will present an unique opportunity for characterizing this object, serving both science and mitigation purposes. The object will be easily visible from the Earth and it can be expected that its shape and thermal properties will be well determined from ground based observations. However, the characterization of its interior will not be achievable from purely terrestrial observations. Such a characterization, beyond its high scientific value, is essential for planning any mitigation operation, should it be necessary in the future.

Near Earth objects are a precious source of information as they represent a mixture of different populations of small bodies containing fundamental information on the origin and early evolution of the solar system.

Monitoring the response of Apophis to the gravitational constraints induced by its close approach to the Earth may provide a way to access information on its internal structure.

A study to identify some affordable mission scenarii for such a mission is presently underway at CNES (the French Space Agency).
\end{abstract}


We will present the scientific and mitigation objectives of such a mission as well as the preliminary results of the mission analysis and the main system characteristics.

Keywords. celestial mechanics, space vehicles: instruments, minor planets, asteroids, etc. 